\title{
Impact of three methods of ischemic preconditioning on ischemia-reperfusion injury in a pig model of liver transplantation
}

\author{
Alessandro Rodrigo Belon \\ Universidade de Sao Paulo \\ Ana Cristina Aoun Tannuri \\ Universidade de Sao Paulo \\ Daniel Albuquerque Moreira \\ Universidade de Sao Paulo \\ Alessandra Matheus Silva \\ Universidade de Sao Paulo \\ Suellen Serafini \\ Universidade de Sao Paulo \\ Raimundo Renato Guimarães \\ Universidade de São Paulo \\ Caroline Silverio Faria \\ Universidade de Sao Paulo \\ Alcione Sanches Alexandre \\ Universidade de Sao Paulo \\ Josiane de Oliveira Gonçalves \\ Universidade de Sao Paulo \\ Vitor Ribeiro Paes \\ Universidade de Sao Paulo \\ Uenis Tannuri ( $\nabla$ uenist@gmail.com) \\ Universidade de Sao Paulo
}

Research article

Keywords: Ischemic preconditioning, Reperfusion injury, Intermittent clamping, Liver transplantation, Remote ischemic preconditioning

Posted Date: April 2nd, 2020

DOI: https://doi.org/10.21203/rs.3.rs-19511/v1

License: (1) (1) This work is licensed under a Creative Commons Attribution 4.0 International License. Read Full License 


\section{Abstract}

Background. Ischemic preconditioning (IPC), either direct (DIPC) or remote (RIPC), is a procedure aimed at reducing the harmful effects of ischemia-reperfusion injury. Aims. To assess the local and systemic effects of DIPC, RIPC, and both combined, in the pig liver transplant model.

Methods. Twenty-four pigs underwent orthotopic liver transplantation and were divided into 4 groups according to the procedures applied: direct donor preconditioning; indirect preconditioning at the recipient and a group with direct donor and indirect recipient preconditioning. The following parameters were recorded: donor and recipient weight, graft-to-recipient weight ratio (GRWR), surgery time, hot and cold ischemia time, and intraoperative hemodynamic values. Blood samples were collected before native liver removal $(\mathrm{BL})$ and at $0 \mathrm{~h}, 1 \mathrm{~h}, 3 \mathrm{~h}, 6 \mathrm{~h}, 12 \mathrm{~h}, 18 \mathrm{~h}$, and $24 \mathrm{~h}$ post-reperfusion and the following biochemical tests were performed: aspartate aminotransferase (AST), alanine aminotransferase (ALT), alkaline phosphatase (ALP), gamma-glutamyl transferase (GGT), creatinine, BUN, lactate, total and direct bilirubin. Histopathological examination of liver, gut, kidney, and lung fragments were performed, as well as molecular analyses for expression of the apoptosis-related BAX (pro-apoptotic) and Bcl-XL (anti-apoptotic) genes, eNOS (endothelial nitric oxide synthase) gene, and IL-6 gene related to inflammatory ischemia-reperfusion injury, using real-time polymerase chain reaction (RT-PCR).

Results. There were no differences between the groups regarding biochemical and histopathological parameters. We found a reduced ratio between the expression of the pro-apoptotic BAX gene and the expression of the anti-apoptotic $\mathrm{Bcl}$ gene in the livers of animals with IPC versus the control group.

Conclusions. DIPC, RIPC or a combination of both produce local beneficial effects only at the molecular level but do not translate into biochemical or histological changes.

\section{Background}

Primary graft dysfunction due to ischemia-reperfusion (I/R) injury is one of the most serious complications of liver transplantation. ${ }^{1,2}$ Ischemic preconditioning (IPC) is an important approach to reduce the harmful effects of this process. It consists of producing brief periods of ischemia followed by brief periods of reperfusion prior to the prolonged ischemic insult, which should induce greater organ tolerance to prolonged ischemia and subsequent reperfusion. ${ }^{3,4}$ Its beneficial effects are thought to result from the release of adenosine by the ischemic tissue promoting vasodilation, inhibition of platelet aggregation and neutrophil adherence, inhibition of endothelin synthesis and reactive oxygen species, in addition to increased production of nitric oxide. ${ }^{4}$

IPC can be direct at the target organ or indirect (remote). Direct ischemic preconditioning (DIPC) has the disadvantage of causing mechanical stress to the main vascular structures of the organ. ${ }^{5}$ In remote ischemic preconditioning (RIPC), the procedure is applied to another organ, with the protective effect on the target organ being exerted by biochemical mediators activated at a distance and carried by the blood stream, without direct stress or trauma to the organ. ${ }^{6}$ The effect of RIPC was first demonstrated on the myocardium of rats submitted to renal IPC. ${ }^{5}$ So far, there is no consensus about the best method of RIPC, i.e., the number of I/R cycles, the effective I/R time required to trigger the protective stimulus, and the choice of the I/R site to maximize the beneficial effects of IPC with the least possible damage to the body. However, notwithstanding these unanswered questions, the short-term occlusion of the mesenteric artery has been proven of great importance, with positive effects on several organs. ${ }^{5}$

Several studies demonstrate the beneficial effects of DIPC and RIPC. A recent study also showed that the combination of both types of IPC enhanced the protective effects of this procedure in a mouse liver transplant model. ${ }^{7}$

The pig model has clear anatomical, morphological, and physiological similarities with humans. It also allows for easy and extensive monitoring and ensures the feasibility of the orthotopic liver transplantation, thus being useful to investigate issues that have direct clinical relevance. ${ }^{7,8}$ The potential benefits of RIPC in transplants performed on medium-sized animals, the association of direct and remote ischemic preconditioning, and the comparative effects of these two methods have been reported in very few studies. Thus, the objectives of the present study were to evaluate the local and systemic effects of DIPC, RIPC and their combination in a liver transplant model in pigs of similar weights. The groups were evaluated by biochemical, histopathological and molecular analyses.

\section{Methods}


The experimental study protocol was approved by the Institutional Animal Use Ethics Committee. All procedures performed in the animals were in accordance with the ethical standards of our Institution. Twenty-four hybrid pigs of both sexes weighing on average $28.7 \pm 2.4 \mathrm{~kg}$ were randomly divided into the following 4 groups $(n=6$ each) and submitted to orthotopic liver transplantation. The number of animals in each group was based in previous similar studies. ${ }^{9,10}$

1. control group (C)

2. direct donor preconditioning (D)

3. indirect recipient preconditioning $(\mathrm{R})$

4. direct donor and indirect recipient preconditioning $(D+R)$.

The animals (donors and recipients) were fasted for 12 hours, then at 7 a.m. injected with intra muscular xylazine $(2.0 \mathrm{mg} / \mathrm{Kg})$ and ketamine $(10.0 \mathrm{mg} / \mathrm{Kg})$ as pre-anesthetic 15 minutes before anesthesia, which was induced with propofol $(5.0-10.0 \mathrm{mg} / \mathrm{Kg}) \mathrm{and}$ maintained with endotracheal intubation, $40 \%$ oxygen supply, and isoflurane (1.3 to $2.0 \%)$ in inspired air, along with a continuous intravenous infusion of fentanyl $(0.05 \mu \mathrm{g} / \mathrm{Kg} / \mathrm{min})$. Catheters were introduced into the jugular vein for fluid infusion and central venous pressure (CVP) measurement, and into the carotid artery for invasive mean arterial pressure (iMAP) measurement and blood sampling for biochemical analyses. Recipient animals were continuously monitored until the end of the surgery and post-surgery recovery with electrocardiogram, oximetry, end tidal carbon dioxide monitoring (EtCO2), respiratory rate, and pressure measurements - CVP and iMAP.

According to the groups where they were allocated, the animals underwent one of two types of ischemic preconditioning (groups $D$ and $\mathrm{R}$ ) or both combined (group $\mathrm{D}+\mathrm{R}$ ). Direct organ preconditioning was performed by clamping the donor whole hepatic pedicle (portal vein, hepatic artery, and bile duct); indirect preconditioning was applied to the recipient gut, by clamping the superior mesenteric artery. Both types of preconditioning consisted of three cycles with 5 minutes' ischemia followed by 5 minutes' reperfusion.

Surgical procedures on donor and recipient animals were performed in the Laboratory of Experimental Surgery (LIM-26) according to previously published technique by our laboratory. ${ }^{9,10}$

After surgery, recipient animals remained extubated and conscious in private stalls in our laboratory for 24 hours, with catheters in the jugular vein and carotid artery for medication infusion and blood sampling. Blood samples were collected at the following time points: before native liver removal (BL) and periods after graft reperfusion: $0 \mathrm{~h}, 1 \mathrm{~h}, 3 \mathrm{~h}, 6 \mathrm{~h}, 12 \mathrm{~h}, 18 \mathrm{~h}$, and $24 \mathrm{~h}$. At the end of this period, the animals were anesthetized, intubated and connected to the mechanical ventilator for gut, kidney, lung, and liver biopsies. After that, the animals were euthanized with an overdose of inhaled anesthetic $5 \%$ isoflurane and intravenous administration of $10 \mathrm{~mL} / \mathrm{kg}$ of $19.1 \%$ potassium chloride.

The following parameters were recorded: donor and recipient weight, graft-to-recipient weight ratio (GRWR), surgery time, hot and cold ischemia time, and intraoperative hemodynamic values. Additionally, the following biochemical tests were performed: aspartate aminotransferase (AST), alanine aminotransferase (ALT), alkaline phosphatase (ALP), gamma-glutamyl transferase (GGT), creatinine, BUN, lactate, total and direct bilirubin.

Histopathological analyses were performed on 4 liver fragments obtained at the following time points: before removal from the donor $(\mathrm{BL})$, immediately after release of the hepatic artery flow of the recipient (0h), after 1 hour of reperfusion ( $1 \mathrm{~h}$ ) and after 24 hours of reperfusion (24h). For the other organs (gut, kidney, and lung), only one biopsy was performed after 24 hours of observation. Samples were fixed in neutral formalin, dehydrated and embedded in paraffin blocks, then sectioned ( $4 \mu \mathrm{m}$-sections) and stained with hematoxylin-eosin.

The liver injury was assessed based on endpoints analyzed and quantified according to the criteria described by Scheuer et al ${ }^{11}$. Lung histology assessment was based on the modified VILI score, with 4 parameters evaluated and quantified to measure the degree of tissue damage ${ }^{12}$. Kidney injury was assessed using the Banff score with 4 parameters evaluated and quantified ${ }^{13}$. To assess histological damage to the gut, the Chiu score was used. ${ }^{14}$

All the tissue sections were examined under light microscopy by 3 different blind readers who assigned scores to the identified injuries. For the statistical analysis, each section was assigned the average of these 3 scores. 
For the molecular analyses, a fragment from each biopsy specimen was examined for expression of the apoptosis-related genes, i.e., BAX (pro-apoptotic) and Bcl-XL (anti-apoptotic), the eNOS (endothelial nitric oxide synthase) gene, and the IL-6 gene related to the inflammatory ischemia-reperfusion injury, using RT-PCR. To study the balance between pro- and anti-apoptotic gene expression, the $\mathrm{BAX} / \mathrm{Bcl}-\mathrm{XL}$ ratio for all organs was calculated at different time points.

\section{Statistical Analysis}

Data were recorded and stored on a spreadsheet of the Stata statistical package. For the qualitative variables, the absolute and relative frequencies were calculated. For quantitative variables, the mean, standard deviation, median, minimum and maximum values were calculated and displayed in graphic format with the values.

Data with normal distribution regarding quantitative variables and gene expression were assessed using analysis of variance (ANOVA); differences between groups were identified using the Bonferroni test; and differences between timepoints in the groups were identified using the t-test. Histomorphometric (qualitative) data were compared using the nonparametric Kruskall-Wallis method.

The null hypothesis of equality of means was rejected when $p<0.05$.

\section{Results}

The recipient weights, GRWR and ischemia time in different groups are presented in Fig. 1. It was observed that there was no difference between the groups with regard to these parameters $(p>0.05)$.

\section{Biochemical Analysis}

The behavior of the different serum biochemical markers studied in the different groups throughout the experimental period is shown in Fig. 2.

Regarding serum AST levels, all groups showed similarly disperse patterns, with values increasing over the experimental time points and stabilizing between 12 and 24 hours. There was no difference between groups.

For gamma-GT, some groups showed great variation within the group at the experimental time points, but no difference was observed between groups. Also, alkaline phosphatase levels showed no differences between groups over the experimental time points.

All groups showed an increase in creatinine values compared to baseline. At $3 \mathrm{~h}$ and $6 \mathrm{~h}$, values in the $\mathrm{R}$ group were lower than in controls $(p=0.003$ and $p=0.042$, respectively).

There was no difference in arterial lactate levels between groups during the experimental period. All groups showed a consistent increase in the immediate post-transplant period, followed by a return, by the end of the experiment, to baseline values.

There was wide variation in total bilirubin levels in the $R$ and $D+R$ groups at $6 h, 12 h, 18 h$, and $24 h$, but the only significant difference observed in this variable was an increase in the $D+R$ group compared to the controls at $24 h(p=0.046)$. The direct bilirubin levels showed the same behavior as total bilirubin, i.e. wide variation within the groups, but with higher levels in the $D+R$ group compared to the controls at $6 \mathrm{~h}(\mathrm{p}=0.033)$.

\section{Histopathological Analysis}

The histopathological analysis of the liver revealed typical ischemia-reperfusion lesions found in major surgeries: lobular and inflammatory cells infiltrate, hepatic sinusoid hyperemia and hepatocellular necrosis. In the small intestine, cell lysis, Grünhagen spaces formation, enlargement of the distance between villi, dilated capillaries, presence of inflammatory cells, and destruction of the villi with intraluminal hemorrhage were observed. In the kidney, the typical lesions were capillary tuft retraction plus interstitial, tubule and vessel congestion. In the lung, alveolar congestion was observed and in some cases hemorrhage and leukocyte infiltrates inside the alveoli and bronchi (Fig. 3). Histopathological scores in the different tissues and groups are summarized in Table 1. Despite individual observations, no statistically significant differences were found in tissue scores between the groups. 
Table 1

Median scores, with maximum and minimum values, of histopathological analyses of different tissues (liver, gut, lung, and kidney), by group and time points (BL, $0 \mathrm{~h}, 1 \mathrm{~h}$, and $24 \mathrm{~h}$ ).

\begin{tabular}{|c|c|c|c|c|c|c|c|c|c|c|c|c|c|}
\hline Group & Time & Liver & & & Gut & & & Kidney & & & Lung & & \\
\hline & & Median & Max & Min & Median & Max & Min & Median & Max & Min & Median & Max & Min \\
\hline \multicolumn{14}{|l|}{ C } \\
\hline & $\mathrm{BL}$ & 1 & 2 & 1 & & & & & & & & & \\
\hline & TOh & 2 & 2 & 1 & & & & & & & & & \\
\hline & T1h & 3 & 3 & 1 & & & & & & & & & \\
\hline & T24h & 3 & 3 & 2 & 0.5 & 3 & 0 & 2 & 2 & 2 & 3 & 4 & 1 \\
\hline \multicolumn{14}{|l|}{ D } \\
\hline & $\mathrm{BL}$ & 1 & 2 & 0 & & & & & & & & & \\
\hline & TOh & 1.5 & 2 & 1 & & & & & & & & & \\
\hline & T1h & 2 & 5 & 2 & & & & & & & & & \\
\hline & T24h & 4 & 7 & 4 & 2.5 & 3 & 0 & 2 & 3 & 0 & 2 & 5 & 0 \\
\hline \multicolumn{14}{|l|}{$\mathrm{R}$} \\
\hline & $\mathrm{BL}$ & 0.5 & 2 & 0 & & & & & & & & & \\
\hline & TOh & 2 & 2 & 1 & & & & & & & & & \\
\hline & T1h & 2 & 2 & 1 & & & & & & & & & \\
\hline & T24h & 3 & 4 & 2 & 0 & 1 & 0 & 1.5 & 4 & 0 & 2 & 3 & 1 \\
\hline \multicolumn{14}{|l|}{$D+R$} \\
\hline & $\mathrm{BL}$ & 0.5 & 2 & 0 & & & & & & & & & \\
\hline & TOh & 2 & 3 & 1 & & & & & & & & & \\
\hline & T1h & 2 & 2 & 2 & & & & & & & & & \\
\hline & T24h & 2.5 & 4 & 2 & 0.5 & 3 & 0 & 2 & 3 & 1.0 & 3.5 & 6 & 2 \\
\hline
\end{tabular}

\section{Molecular Analysis}

The gene expressions in the liver, gut, kidney, and lung of the study animals are shown in Fig. 4. In the liver, concerning the BAX gene expression, the $D+R$ group showed lower values at $24 \mathrm{~h}$ when compared to the controls $(p=0.039)$. The expression of the IL-6 gene in $D$ group animals was higher at $24 \mathrm{~h}$ when compared to the $R$ group $(p=0.001)$ and the $D+R$ group $(p=0.02)$. The $B c l-X L$ values did not increase during the experimental period in the controls, while in the groups that received any preconditioning there was an increase in the gene expression towards the end of the experimental period; the $D$ group and the $D+R$ group showed higher values at $24 h$ ( $p=$ 0.034 and $p=0.006$, respectively) when compared to the controls. Regarding the e-NOS gene, by $24 \mathrm{~h}$ the values of the $D+R$ were higher when compared to the controls $(p=0.031)$, the $D$ group $(p=0.003)$, and the R group $(p=0.021)$.

In the gut, no differences in the expression of the BAX and IL-6 genes were observed in the groups at $24 \mathrm{~h}$. However, the Bcl-XL gene expression was higher in the R group than in the controls $(p=0.004)$. In addition, the eNOS gene expression was higher in the $D$ group animals when compared to the controls $(p=0.001)$ and the R group $(p=0.001)$. In the kidney, it was observed that there was no difference in the expression of the BAX and Bcl-XL genes in the various groups at $24 \mathrm{~h}$. IL- 6 in the R group showed higher values than in the controls $(p=0.004)$. Also, no difference between groups in the expression of the BAX, IL-6 and Bcl-XL genes in the lung tissue, although the eNOS gene expression was higher in the $D$ group than in the $R$ group $(p=0.007)$. Finally, the ratio of pro-apoptotic $B A X$ gene expression to anti-apoptotic $B c l-X L$ gene expression was lower in the liver by $24 h$ in all groups receiving IPC (D, R, and $D+R$ groups) when compared to the controls (Fig. 5). 


\section{Discussion}

While many studies demonstrate the benefits of ischemic preconditioning in liver resection surgeries of small animals, ${ }^{15-18}$ there are few studies with models of cold and hot ischemia in liver transplantation performed on medium-sized animals. ${ }^{19,20}$

In previous studies, we have used a model similar to the present one and observed that IPC resulted in partial attenuation of the harmful effects of $\mathrm{I} / \mathrm{R}$ injury.10 In the current study, we also aimed at identifying if the positive effects of IPC remained after a longer observation time, thus providing a rationale for its use in clinical practice.

While studies of IPC are not new, still there is no consensus about technical issues such as ischemia time and number of $\mathrm{I} / \mathrm{R}$ cycles needed to effectively achieve protective effects. Therefore, in our model, we used 3 alternating 5 -minute cycles of ischemia followed by the same reperfusion time, to prevent severe hemodynamic repercussions in the animals, while avoiding a too short IPC period that might have less evident effects. Another objective of this experimental study was to assess RIPC as a means to protect the target organ without causing the direct stress of ischemia-reperfusion. By using the two techniques in separate groups as well as combined, in a specific group that received the two types of IPC, our goal was to ascertain if there would be a difference between preconditioning the graft or the recipient, and to check potential cumulative effects when both procedures are used concomitantly.

For RIPC, the most widely used technique is clamping one limb of a patient ${ }^{21}$ or animal. ${ }^{22}$ However, for our project we chose a different target territory, i.e. the gut, by clamping the superior mesenteric artery. This organ was chosen because it has one of the highest levels of metabolic activity and is very susceptible to oxygen pressure variations, and therefore is quickly responsive to short periods of ischemia, which should maximize the protective effect of the RIPC. Finally, it is important to stress that the gut is a territory that markedly suffers from blood stasis during the anehepatic phase of the transplant procedure.

We performed the current experiments to clarify local and systemic effects of IPC in liver transplantation and assess the potential influence of our conditioning models on common problems caused by I/R injury in organ transplants, such as acute kidney injury. ${ }^{23-25}$ Unlike previous publications, our biochemical results fail to show any benefit of DIPC or RIPC in liver transplantation. Serum AST and ALT levels are consistent with the degree of hepatocellular injury and are used as indicators of graft distress, bearing correlation with different levels of primary graft dysfunction. All groups in our study were comparable in terms of enzymatic profile. In the $D+R$ group, AST showed high variability, with a trend towards higher median values at $12 \mathrm{~h}, 18 \mathrm{~h}$, and $24 \mathrm{~h}$, suggesting a possible harmful effect of the addition of the two IPC procedures, even though the difference was not significant.

Our results differ from those of studies of liver transplantation in small animals (rats), which showed lower transaminase values and improvement of histopathological aspects in animals submitted to RIPC compared to controls after $24 \mathrm{~h}$ of reperfusion.23 On the other hand, our results are consistent with those from human studies in which direct and remote IPC increased AST and ALT values $24 \mathrm{~h}$ after reperfusion. ${ }^{21,26}$

However, the molecular analysis has shown some beneficial effects of IPC. In the liver tissue, the combined IPC approach resulted in marked positive changes in gene expression. The eNOS gene expression in the liver tissue was higher in the $D+R$ group at $24 \mathrm{~h}$, and such expression is usually related to improvement in ischemia. In addition, lower expression of pro-inflammatory genes (BAX) and higher expression of anti-inflammatory genes $(\mathrm{Bcl}-\mathrm{XL})$ were observed in this same group. DIPC also had positive effects, leading to increased expression of the IL-6 and Bcl-XL genes.

The BAX/Bcl-XL ratio showed lower values for all treated groups when compared with controls at $24 \mathrm{~h}$. This finding may suggest an IPC-driven potential decrease in cell apoptosis secondary to I/R injury. Furthermore, in the gut, kidney, and lung tissues, some molecular changes were detected demonstrating beneficial effects of each IPC separately.

Although gene expression suggests positive effects, the results of biochemical and histopathological analyses, consistent with human studies, did not confirm any benefits from IPC, which raises questions about the feasibility of extrapolating results obtained in small animals to medium-sized animals and humans. The first hypothesis to explain this difference would be a higher susceptibility of certain species, e.g. rodents, to the IPC procedure, either in situ (direct) or remote, with this propensity diminishing as we advance phylogenetically towards humans, as in the case of pigs.

Also, worth mentioning are the physiological, anatomical, and surgical variations involved in organ transplantation in different species. Despite the highly ingenious technical solutions found to overcome difficulties and enable organ transplantation in rats and mice, the

Page $6 / 13$ 
surgical procedure in these animals cannot match what happens in human surgery, including technical hurdles, hemodynamic instability, surgical time, and postoperative follow-up. The human context is optimally mimicked by transplantation performed in medium-sized animals, which yields similar results.

\section{Conclusions}

In the pig model of orthotopic liver transplantation, direct or remote IPC, or a combination of both produces local beneficial effects only at the molecular level but do not translate into biochemical or histological changes.

\section{Availability Of Data And Materials}

The datasets used and analyzed during the current study will be available from the corresponding author on reasonable request.

\section{Abbreviations}

IPC

Ischemic preconditioning

DIPC

Direct ischemic preconditioning

RIPC

Remote ischemic preconditioning

GRWR

Graft-to-recipient weight ratio

BL

Before native liver removal

AST

aspartate aminotransferase

ALT

alanine aminotransferase

ALP

alkaline phosphatase

GGT

gamma-glutamyl transferase

BUN

blood urea nitrogen

RT-PCR

real-time polymerase chain reaction $\mathrm{BAX}$

CVP

central venous pressure

iMAP

invasive mean arterial pressure

\section{Declarations}

\section{Ethics approval and consent to participate}

The experimental study protocol number 034/14 was approved by the Institutional Animal Use Ethics Committee (University of Sao Paulo Medical School, Sao Paulo, Brazil). All applicable international, national, and institutional guidelines for the care and use of animals were followed. The landrace white pigs were commercially obtained from the Company Granja RG (Suzano - São Paulo, Brazil). A written informed consent to use the animals in the study was obtained from the Company. 
Not applicable

\section{Availability of data and materials}

All data generated or analysed during this study are included in this published article [and its supplementary information files]

\section{Competing interests}

The authors declare that they have no conflict of interest.

\section{Funding}

The laboratory studies of the current investigation were supported by Fundação de Amparo a Pesquisa do Estado de São Paulo (Research number 2014/25676-0).

\section{Author's contribution}

ARB, ACAT, RRG, performed the animal experiments; AMS, SS, JOG, CSF, ASA, VRP performed all laboratory studies; DARM performed the statistical analyses and UT was the responsible for the manuscript.

All authors have read and approved the manuscript.

\section{Acknowledgments}

Not applicable.

\section{References}

1. Liu Q, Bruns H, Schultze D, Xue Y, Zorn M, Flechtenmacher C, Straub BK, Rauen U, Schemmer P. HTK-N, a modified HTK solution, decreases preservation injury in a model of microsteatotic rat liver transplantation. Langenbecks Arch Surg 2012;397:1323-1331.

2. Kupiec-Weglinski JW, Busuttil RW. Ischemia and reperfusion injury in liver transplantation. Transplant Proc. 2005;37:1653-1656.

3. Theodoraki K, Tympa A, Karmaniolou I, Tsaroucha A, Arkadopoulos N, Smyrniotis V. Ischemia/reperfusion injury in liver resection: a review of preconditioning methods. Surg Today 2011;41:620-629.

4. Miranda LEC, Viaro F, Ceneviva R, Evora PRB. As bases experimentais da lesão por isquemia e reperfusão do fígado: revisão. Acta Cirurgica Bras 2004;19:1-12.

5. Tapuria N, Kumar Y, Habib MM, Abu Amara M, Seifalian AM, Davidson BR. Remote ischemic preconditioning: a novel protective method from ischemia reperfusion injury-a review. J Surg Res 2008;150:304-330.

6. Song X, Zhang N, Xu H, Cao L, Zhang H. Combined preconditioning and postconditioning provides synergistic protection against liver ischemic reperfusion injury. Int J Biol Sci 2012;8:707-718.

7. Mendes-Braz M, Elias-Miro M, Jimenez-Castro MB, Casillas-Ramirez A, Ramalho FS, Peralta C. The current state of knowledge of hepatic ischemia-reperfusion injury based on its study in experimental models. J Biomed Biotechnol 2012;2012:298657.

8. Yandza T, Tauc M, Saint-Paul MC, Ouaissi M, Gugenheim J, Hebuterne X. The pig as a preclinical model for intestinal ischemiareperfusion and transplantation studies. J Surg Res 2012;178:807-819. 
9. Rangel Moreira D de A, Aoun Tannuri AC, Belon AR, Mendonça Coelho MC, Oliveira Gonçalves J, Serafini S, Roberto Lima F, Agostini LO, Guimarães RR, Tannuri U. Large-for-size liver transplantation: a flowmetry study in pigs. J Surg Res. 2014;189:313320.

10. Leal AJ, Tannuri AC, Belon AR, Guimarães RR, Coelho MC, Gonçalves J de O, Serafini S, Melo ES, Tannuri U. Effects of ischemic preconditioning in a pig model of large-for-size liver transplantation. Clinics (Sao Paulo) 2015;70(2):126-135.

11. Scheuer PJ, Standish RA, Dhillon AP. Scoring of chronic hepatitis. Clin Liver Dis 2002;6:335-347.

12. Belperio JA, Keane MP, Burdick MD, Londhe V, Xue YY, Li K, Phillips RJ, Strieter RM. Critical role for CXCR2 and CXCR2 ligands during the pathogenesis of ventilator-induced lung injury. J Clin Invest 2002;110:1703-1716.

13. Racusen LC, Halloran PF, Solez K. Banff 2003 meeting report: new diagnostic insights and standards. Am J Transplant 2004 Oct;4(10):1562-6. PubMed PMID:15367210.

14. Chiu CJ, McArdle AH, Brown R, Scott HJ, Gurd FN. Intestinal mucosal lesion in low-flow states. I. A morphological, hemodynamic, and metabolic reappraisal. Arch Surg 1970;101:478-483.

15. Gomes PFM, Tannuri ACA, Nogueira TM, luamoto LR, Paes VR, Coelho MCM, Gonçalves JO, Serafini S, Tannuri U. Remote Ischemic Preconditioning Is Efficient in Reducing Hepatic Ischemia-Reperfusion Injury in a Growing Rat Model and Does Not Promote Histologic Lesions in Distant Organs. Transplant Proc 2018;50:3840-3844.

16. Koti RS, Seifalian AM, McBride AG, Yang W, Davidson BR. The relationship of hepatic tissue oxygenation with nitric oxide metabolism in ischemic preconditioning of the liver. FASEB J 2002;16:1654-1656.

17. Koti RS, YangW, DashwoodMR, DavidsonBR, Seifalian AM. Effect of ischemic preconditioning on hepatic microcirculation and function in a rat model of ischemia reperfusion injury. Liver Transpl 2002; 8:1182-1191.

18. Gustafsson BI, Friman S, Wallin M, Heiman J, Delbro DS. Effect of remote preconditioning on mild or severe ischemia-reperfusion injury to rat liver. Transplant Proc 2006;38:2708-2709.

19. Shimoda M, Iwasaki Y, Sawada T, Kubota K. Protective effect of ischemic preconditioning against liver injury after major hepatectomy using the intermittent Pringle maneuver in swine. Pathobiology 2007;74:42-49.

20. Przyklenk K, Bauer B, Ovize M, Kloner RA, Whittaker P. Regional ischemic 'preconditioning' protects remote virgin myocardium from subsequent sustained coronary occlusion. Circulation 1993;87:893-9.

21. Robertson FP, Goswami R, Wright GP, Imber C, Sharma D, Malago M, Fuller BJ, Davidson BR. Remote ischaemic preconditioning in orthotopic liver transplantation (RIPCOLT trial): a pilot randomized controlled feasibility study. HPB (Oxford) 2017;19:757-767.

22. Czigany Z, Bleilevens C, Beckers C, Stoppe C, Möhring M, Fülöp A, Szijarto A, Lurje G, Neumann UP, Tolba RH. Limb remote ischemic conditioning of the recipient protects the liver in a rat model of arterialized orthotopic liver transplantation. PLoS One 2018;13:e0195507.

23. Liu X, Cao L, Zhang T, Guo R, Lin W. Effect of Remote Ischemic preconditioning in patients undergoing hepatectomy with portal triad clamping: A randomized controlled trial. Anesth Analg 2019;129:1742-1748.

24. Barri YM, Sanchez EQ, Jennings LW, Melton LB, Hays S, Levy MF, Klintmalm GB. Acute kidney injury following liver transplantation: definition and outcome. Liver Transpl 2009;15:475-483.

25. Yalavarthy R, Edelstein CL, Teitelbaum I. (2007) Acute renal failure and chronic kidney disease following liver transplantation. Hemodial Int 11:S7-S12.

26. Koneru B, Shareef A, Dikdan G, Desai K, Klein KM, Peng B, Wachsberg RH, de la Torre AN, Debroy M, Fisher A, Wilson DJ, Samanta AK. The ischemic preconditioning paradox in deceased donor liver transplantation-evidence from a prospective randomized single blind clinical trial. Am J Transplant 2007;7:2788-2796.

\section{Figures}



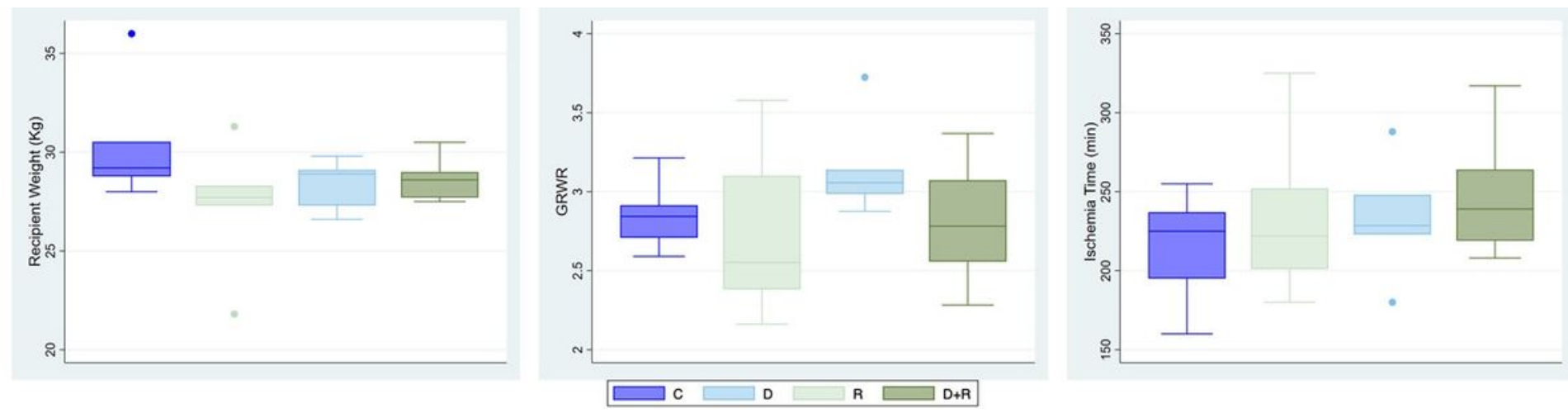

\section{Figure 1}

Recipient weight, GRWR and ischemia time in different groups. C: control group; D: direct donor preconditioning group; R: indirect recipient preconditioning group; and $\mathrm{D}+\mathrm{R}$ : direct donor and indirect recipient preconditioning group. $\mathrm{p}>0.05$ for all comparisons. Note the outliers.
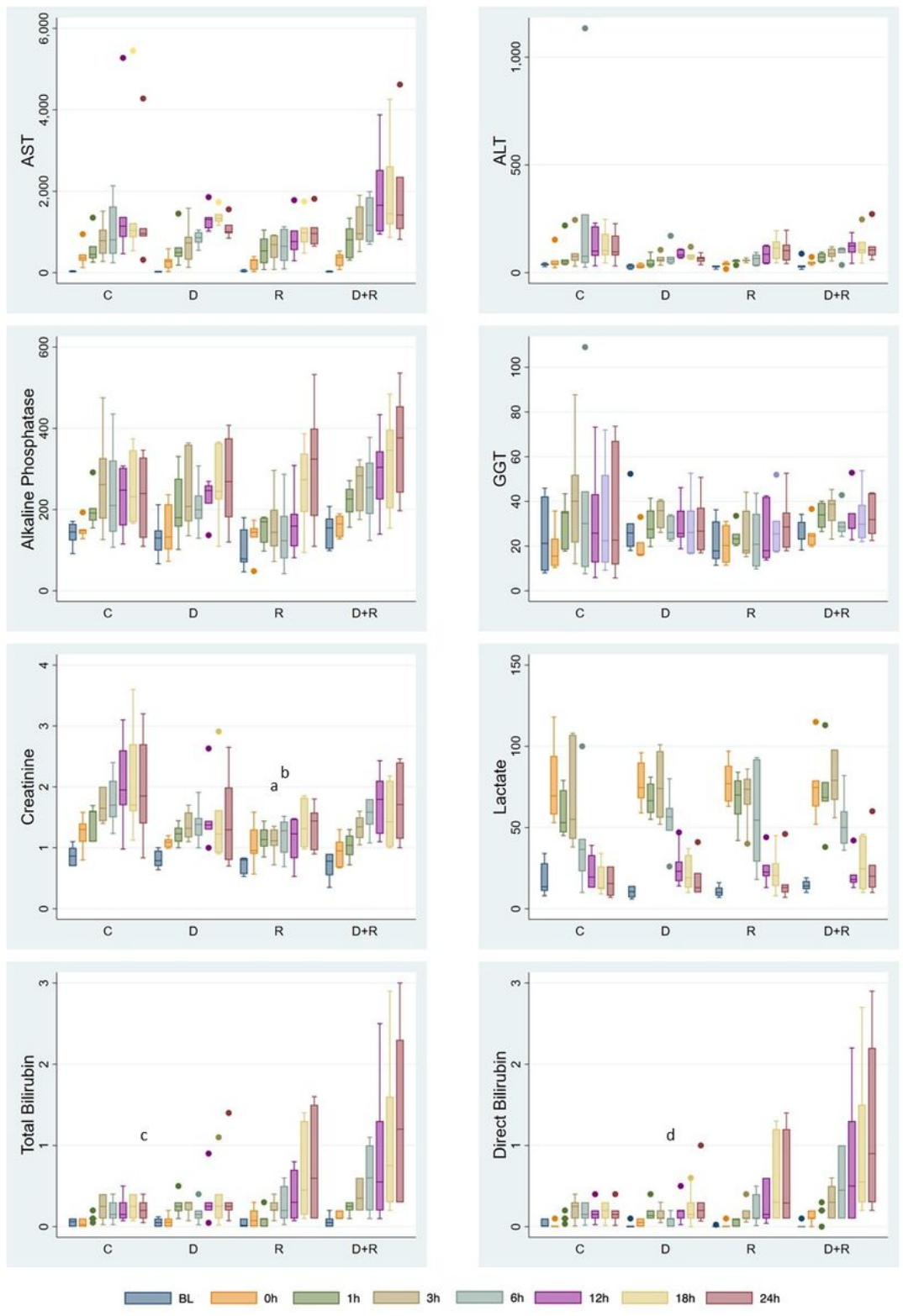

Figure 2 
Measurements of blood biochemical parameters, in different groups by time point. Creatinine: $C$ group vs. $R$ group, $p=0.003 ; b$ : $C$ group vs. $D+R$ group, $p=0.040$. Total bilirubin: $D$ group vs. $D+R$ group, $p=0.046$. Direct bilirubin: $D$ group vs. $D+R$ group, $p=0.033$.

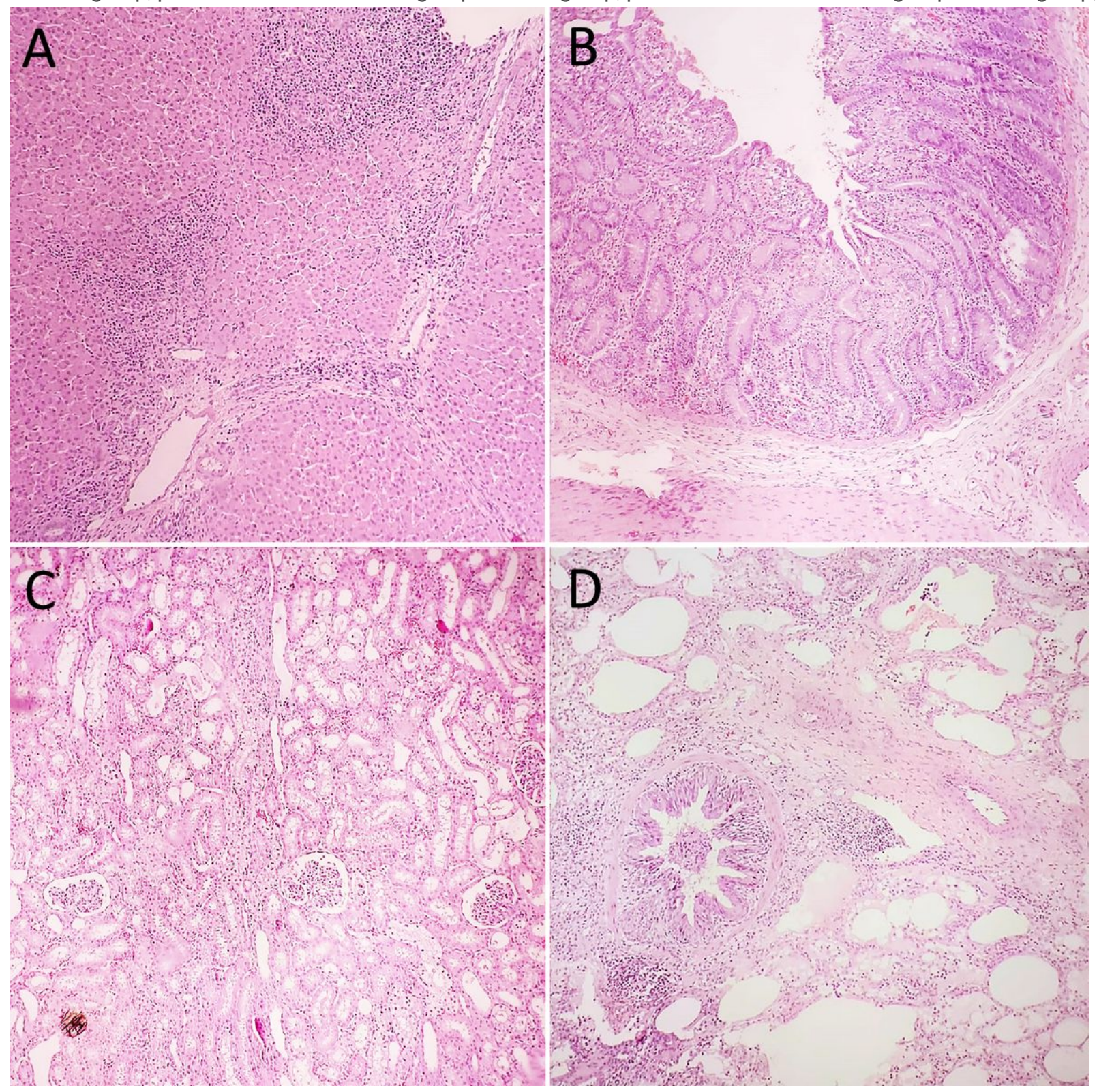

\section{Figure 3}

Histopathological analysis: A: Liver: observe the lobular and inflammatory cells infiltrate; B: Gut: note the inflammatory cells and destruction of the villi; C: Kidney: an intense capillary tuft retraction is observed; $\mathrm{D}$ : Lung: observe the inflammatory cell infiltrate in the alveolar septa and inside the bronchus. 
Liver
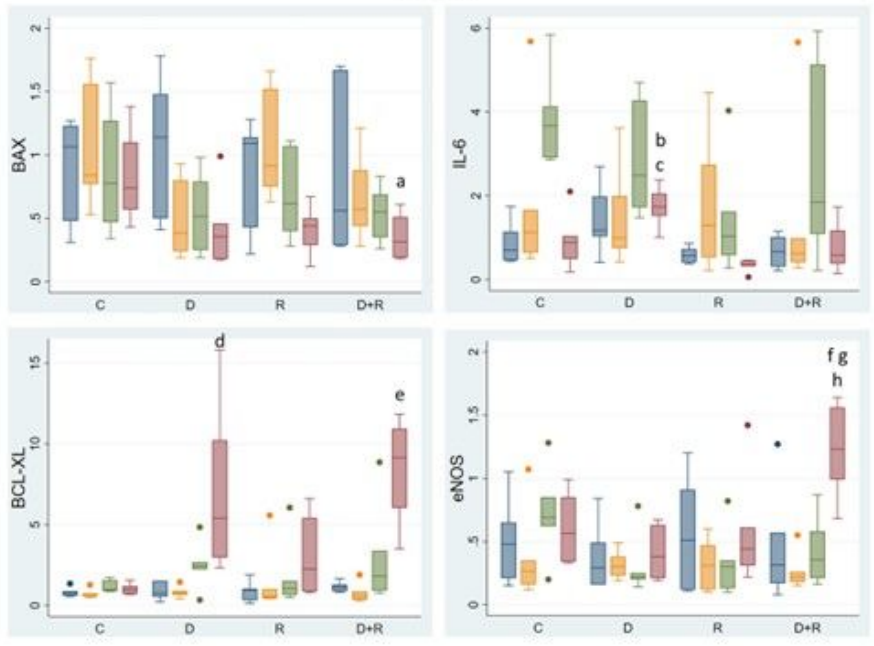

Kidney
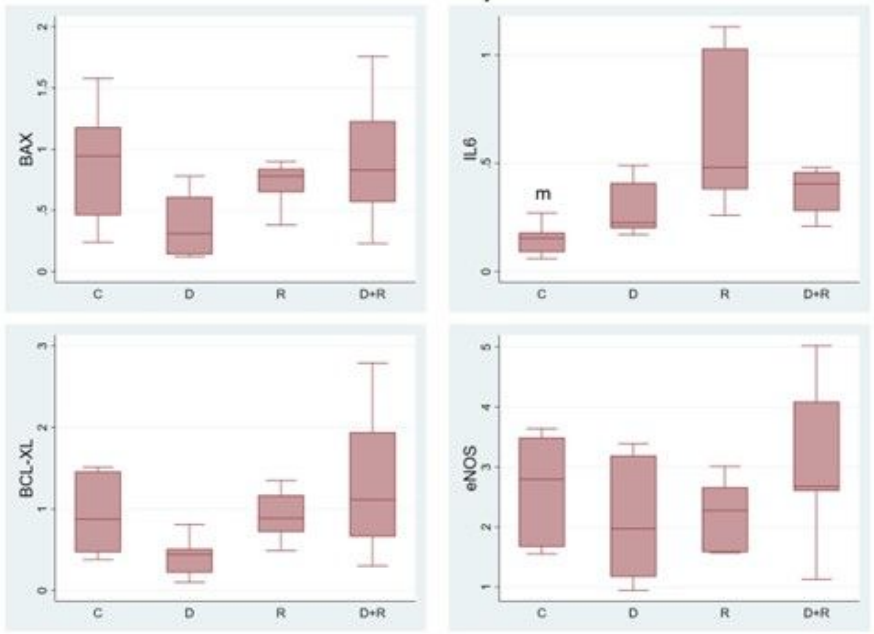

Gut
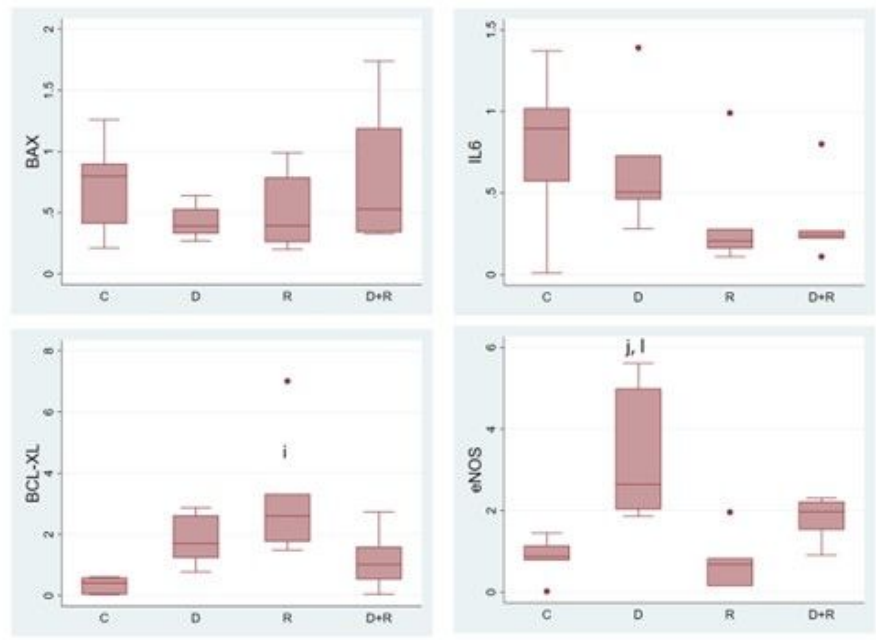

Lung
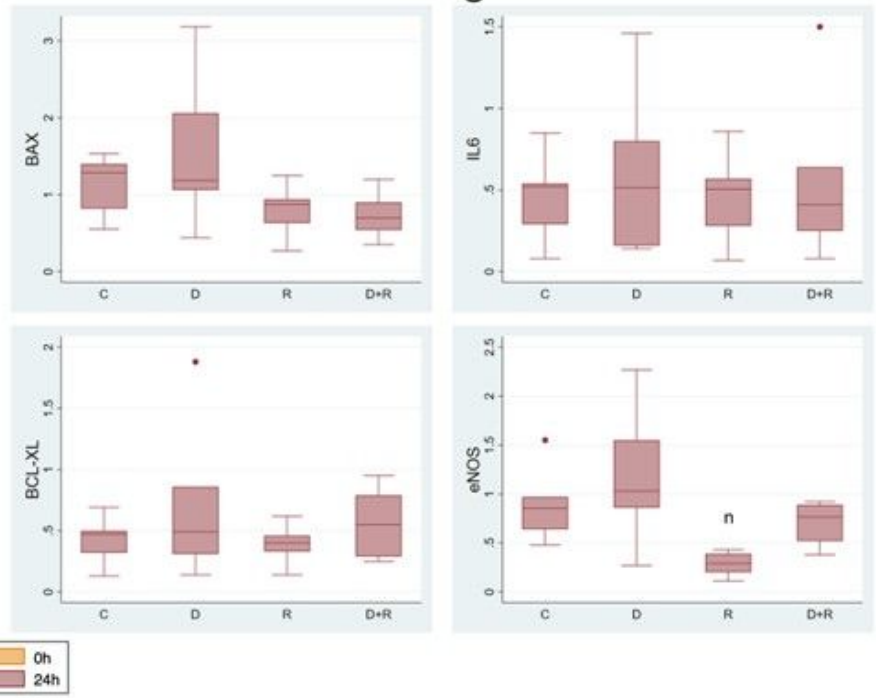

\section{Figure 4}

Expression of BAX, IL-6, Bcl, and e-NOS genes in the liver, gut, kidney, and lung tissues, in different groups by time point. Liver - BAX gene: $C$ group vs. $D+R$ group, $p=0.039$; IL-6 gene: $D$ group vs. $R$ group, $p=0.001 ; D$ group vs. $D+R$ group, $p=0.020$; gene $B C L-X L: C$ group vs. $D$ group, $p=0.034 ; C$ group vs. $D+R$ group, $p=0.006 ;$ eNOS gene: $C$ group vs. $D+R$ group, $p=0.031 ; D$ group vs. $D+R$ group, $p=0.003 ; R$ group vs. $D+R$ group, $p=0,021$. Gut $-B C L-X L$ gene: $C$ group vs. $R$ group, $p=0.004 ;$ eNOS gene: $C$ group vs. $D$ group, $p=0,001 ; D$ group vs. $R$ group, $p=0.001 ;$ Kidney - IL6 gene: $C$ group vs. $R$ group, $p=0.004$. Lung - eNOS gene, $D$ group vs. $R$ group, $p=0.007$. 
BAX/BCL-XL
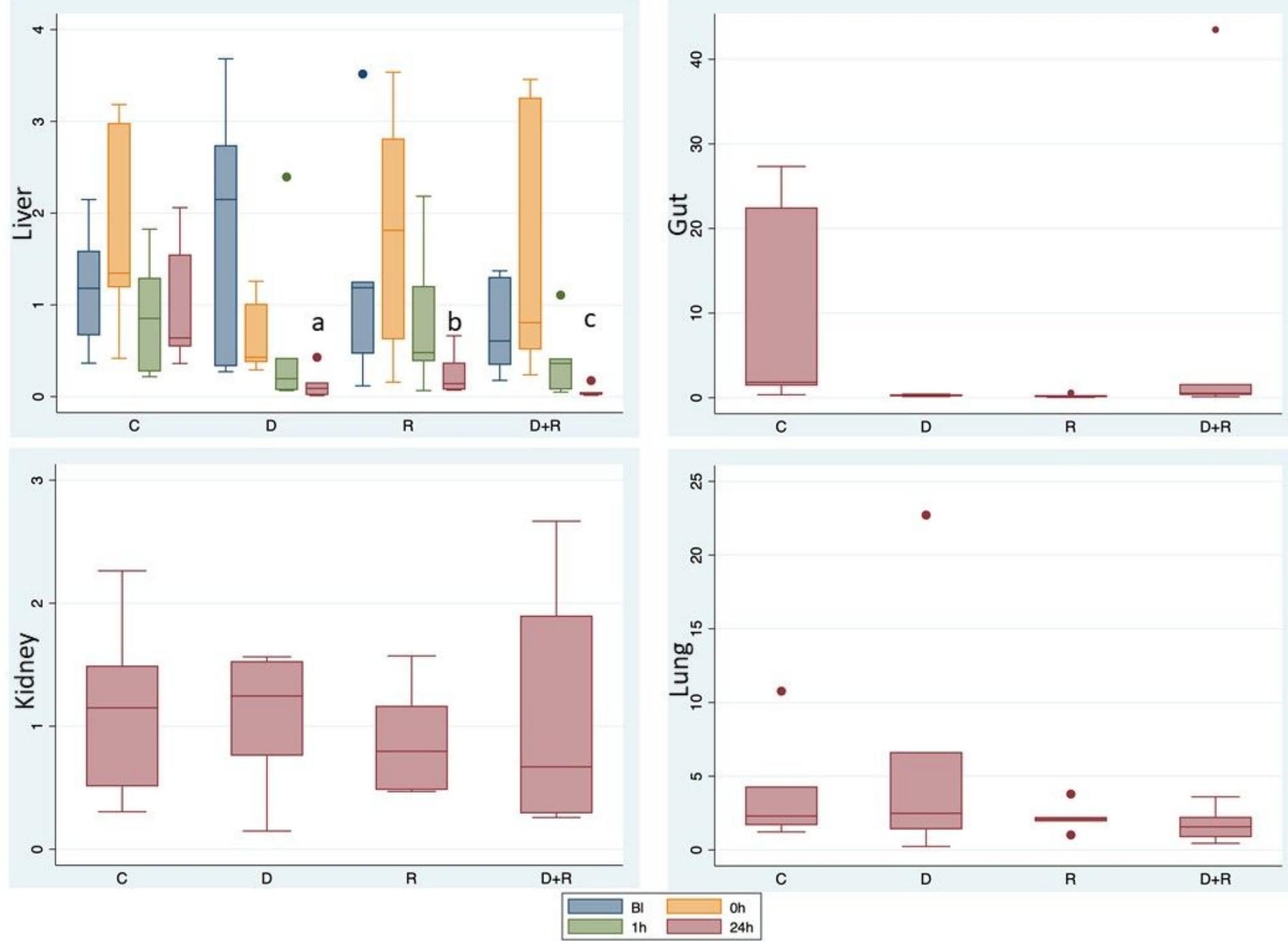

Figure 5

$\mathrm{BAX} / \mathrm{Bcl}$ ratio in the liver, gut, kidney, and lung tissues, in different groups by time point. $\mathrm{C}$ group vs. $\mathrm{D}$ group, $\mathrm{p}=0.005 ; \mathrm{C}$ group vs. $\mathrm{R}$ group, $p=0.017 ; C$ group vs. $D+R$ group, $p=0.002$. Note the outliers.

\section{Supplementary Files}

This is a list of supplementary files associated with this preprint. Click to download.

- arrive.pdf 